\title{
Analysis of Risk Factors and Clinical Indicators in Bloodstream Infections Among Patients with Hematological Malignancy
}

This article was published in the following Dove Press journal: Cancer Management and Research

\author{
Yating $M a^{1,2}$ \\ Shijian Wang ${ }^{2}$ \\ Ming Yang ${ }^{3}$ \\ Jinfeng Bao' \\ Chengbin Wang ${ }^{1,2}$ \\ 'Department of Clinical Laboratory, The \\ PLA General Hospital, Beijing 100853 , \\ People's Republic of China; ${ }^{2}$ Nankai \\ University School of Medicine, Nankai \\ University, Tianjin 30007I, People's \\ Republic of China; ${ }^{3}$ Department of \\ Laboratory Medicine, The Third Xiangya \\ Hospital of Central South University, \\ Changsha 410013, People's Republic of \\ China
}

Purpose: The incidence of bloodstream infection (BSI) is more common in patients with hematological malignancy. It is important to distinguish infectious episodes from noninfectious episodes. The present study was aimed to describe the epidemiology, clinical indexes, and antibiotic use for in-hospital bloodstream infections of hematological malignancy patients.

Patients and Methods: Single-center retrospective research was performed on hematological malignancy patients admitted to our hospital from July 2015 to March 2018. Laboratory and clinical information from 322 febrile patients were acquired. These episodes were divided by blood culture results into two groups: 1) blood culture positive-group, 2) blood culture negative-group.

Results: In the 322 febrile cases, $81(25.2 \%)$ patients were blood culture positive, and among them, Gram-negative (G-) bacteria $(51.9 \%)$ were more isolated than Gram-positive $(\mathrm{G}+)$ bacteria $(32.1 \%)$ and fungi $(7.4 \%)$. Gram-negative bacteria were more likely to have drug resistance than $\mathrm{G}+$ bacteria. Independent risk factors revealed that patients with complications, high levels of procalcitonin (PCT), glucose, interleukin-6 (IL-6), and d-dimer (D-D), and low concentration of albumin were correlated with the occurrence of BSI. PCT, IL-6 and D-D performed well in differentiating the positive group from the negative group. Moreover, IL-6 and D-D showed excellent performance in differentiating $\mathrm{G}-$ and $\mathrm{G}+$ groups, with the areas under the curve all above 0.8 .

Conclusion: We analyzed the risk factors for BSI in patients with hematological malignancy, the distribution of bacteria, antibiotic resistance, and the changes in clinical parameters. This single-center retrospective study may provide clinicians insight into the diagnosis and treatment of BSI.

Keywords: bacteremia, hematological malignancy, risk factors, interleukin-6, D-dimer

\section{Introduction}

Bloodstream infection (BSI), which is defined as laboratory-confirmed isolation of at least one Gram-negative or G+ bacteria or other pathogens from blood samples, ${ }^{1}$ remains a common concern for hematological malignancy patients who often receive chemotherapy or hematopoietic stem cell transplants. ${ }^{2,3}$ Among pathogen distribution, bacterial BSI was more common in the clinic. Distinguishing infectious from noninfectious episodes is more complicated in hematological malignancy patients, for they all had the key manifestation of fever. There are various reasons related to fever, such as pathogen infection, graft-versus-host disease, engraftment syndrome,
Correspondence: Chengbin Wang Email wangcb30I@I26.com 
and thrombotic microangiopathy; thus, early recognition of fever cause is necessary to offer timely antibiotic therapy. ${ }^{4}$ As a result, sufficient and immediate clinical laboratory results are critical for patients with a suspected infection.

Blood culture, the gold standard of infection diagnosis, often takes 3-5 days and is limited by the relatively low positive rate, which restricts its use in early diagnosis. ${ }^{5}$ Patients with infection are often accompanied by changes in biochemical indexes, such as albumin (ALB), glucose (GLU), ${ }^{6,7}$ alanine transaminase (ALT), and aspartate transaminase (AST). Some clinical parameters, including white blood cell (WBC), platelets (PLT), C-reactive protein (CRP), procalcitonin (PCT), ${ }^{8}$ interleukin-6 (IL-6), ${ }^{9}$ and d-dimer (D-D), ${ }^{10}$ are commonly used in diagnosing infection. However, these parameters have not been widely studied in hematological malignancy patients. Therefore, in this study, the clinical characteristics, bacterial distribution, antibiotic use and resistance, inflammation biomarkers, and risk factors of 322 hematologic malignancy patients with high fever were systematically and retrospectively analyzed to predict infection.

\section{Patients and Methods}

\section{Enrollment of Patients and Clinical Data Collection}

This retrospective study was conducted at the Chinese People's Liberation Army (PLA) General Hospital and was approved by the Ethical Committee (No. S2018207,002). The study was carried out as per the Declaration of Helsinki. All adult patients (age $\geq 18$ years old) and diagnosed with hematological malignancies accompanied by febrile syndrome undergoing blood culture were recorded from July 2015 to March 2018. Fever was defined as an axillary body temperature above $37.5^{\circ} \mathrm{C}$. Subjects who had insufficient data or were determined to have a positive blood culture due to contamination were excluded. Samples from a total of 322 patients were analyzed. Only patients who had all clinical and laboratory data available were included, such as WBC, PLT, CRP, PCT, IL-6, D-D, ALB, GLU, ALT, AST, blood culture results, antibiotic use, and resistance. BSI was diagnosed with blood culture results, indicating the types of microorganisms causing the infection. Febrile patients were divided into two groups according to blood culture results: the blood culture-positive group and blood culturenegative group; among blood culture-positive group, it can be divided as culture-positive bacterial infection by
Gram-positive cocci (GPC), culture-positive bacterial infection by Gram-negative bacilli (GNB), culturepositive fungal infection (Fungemia), and others.

\section{Laboratory Tests}

When patients had infectious syndrome, including a high fever, a cough, a chill or hemodynamic instability, blood samples $(5-10 \mathrm{~mL})$ from peripheral intravenous were collected for each blood culture set and divided to inoculate into two blood culture bottles, one for aerobes and another for anaerobes in Bactec Plus Aerobic/F (BD) bottles. Blood samples from indwelling peripherally inserted central venous catheter were not allowed to undergo the blood culture. Moreover, another blood sample was tested to measure WBC, PLT, CRP, PCT, IL-6, D-D, ALB, GLU, ALT, and AST levels. Blood samples were taken before the initiation of empiric antibacterial therapy in all patients. The species of blood culture isolates were recognized by MALDI-TOF-MS (Bio-Merieux, Marcy l'Etoile, France). Antimicrobial susceptibility was tested by a Vitek 2 system (Bio-Mérieux). WBC and PLT were measured with a Sysmex-XN9000 hematology analyzer. Serum CRP concentration was detected using a PuMen CRP analyzer. PCT was tested using a Roche Cobas 8000. ALB, GLU, ALT, and AST were measured in Roche Cobas c501. IL-6 was tested in Roche E411. D-D was determined in a STAGO coagulation analyzer (France).

\section{Statistical Analysis}

Continuous variables are expressed as the mean \pm standard deviation (assessed using Student's $t$-test) or as the median (range) (evaluated using the Wilcoxon rank-sum test) when the distribution was not normal. Categorical variables are expressed as percentages and were analyzed using the Chi-squared test. The strength of associations was determined by calculating the odds ratio (OR) and 95\% confidence intervals (CIs). The independent indices forecasting BSI in hematologic patients with febrile episodes were identified by logistic regression. Any index with $P<0.2$ in univariate analysis was sent to logistic regression. Each index's reliability for bacterial BSI diagnosis was assessed using the receiver-operating characteristic (ROC) curve and area under the curve (AUC). Diagnostic accuracy, including sensitivity and specificity, was computed using cut-off values. The optimal diagnostic cut-off level was found using Youden's index. All analyses were performed on SPSS 19.0 (IBM Corp., Armonk, NY, USA) and GraphPad Prism 6.0 (GraphPad Software, La Jolla, CA, USA). $P<0.05$ implied significance. 


\section{Results}

\section{Clinical Characteristics of Patients with Hematological Malignancies}

A total of 475 blood cultures were collected during the period. Fifty-four patients were removed because they were under 18 years old; 86 patients were excluded due to incomplete data, and 13 because of contamination (Figure 1). In all, 322 samples from high fever patients were analyzed and separated by blood culture results in a blood culture-positive group and a blood culturenegative group. The general characteristics are shown in Table 1. There were more male patients in these two groups. Median ages were 44 and 43 years old, respectively. The main primary diseases of these two groups were acute myeloid leukemia (AML), lymphoma, acute lymphoblastic leukemia (ALL), and myelodysplastic syndrome (MDS). Patients with hematological malignancies were often accompanied by further complications, such as pneumonia, septicemia, cystitis, urinary tract infection, perianal infection, stress ulcer, and others. The data for complications were recorded when the patient presented with symptoms. This was completed during blood collection from hospitalized patients. The blood culture-positive group tended to have more complications $(88.9 \%)$ than non-infected patients (68.9\%). Among them, the only septicemia was significantly different between these two groups $(P<0.001)$. Moreover, there were high percentages of diabetes and smoking in the blood culture-positive group, while there were no differences between them $(P$ values of diabetes and smoking were 0.522 and $0.128)$. There was also not much difference in the length of hospital stay between the two groups $(P=0.891)$ (Table 1).

\section{Distribution of Pathogens and the Use of Antibiotics and Resistance}

The distribution of pathogens is shown in Figure 2. The most frequent G- bacterium in blood culture was Escherichia coli (E. coli) (21\%), followed by Klebsiella pneumoniae (K. pneumoniae) (8.6\%), Pseudomonas aeruginosa (P. aeruginosa) (6.2\%), and Brucella (6.2\%). The frequent $\mathrm{G}+$ bacteria were Staphylococcus hominis (S. hominis) (12.3\%), Staphylococcus epidermidis (S. epidermidis) (7.4\%), Coagulase-negative Staphylococci (CNS) (3.7\%), Staphylococcus capitis (S. capitis) (2.5\%) and Staphylococcus hemolyticus (S. hemolyticus) (2.5\%). Fungemia occupied $7.4 \%$ in the blood culture-positive group; multiple bacterial $(\geq 2)$ infections occurred in $8.6 \%$

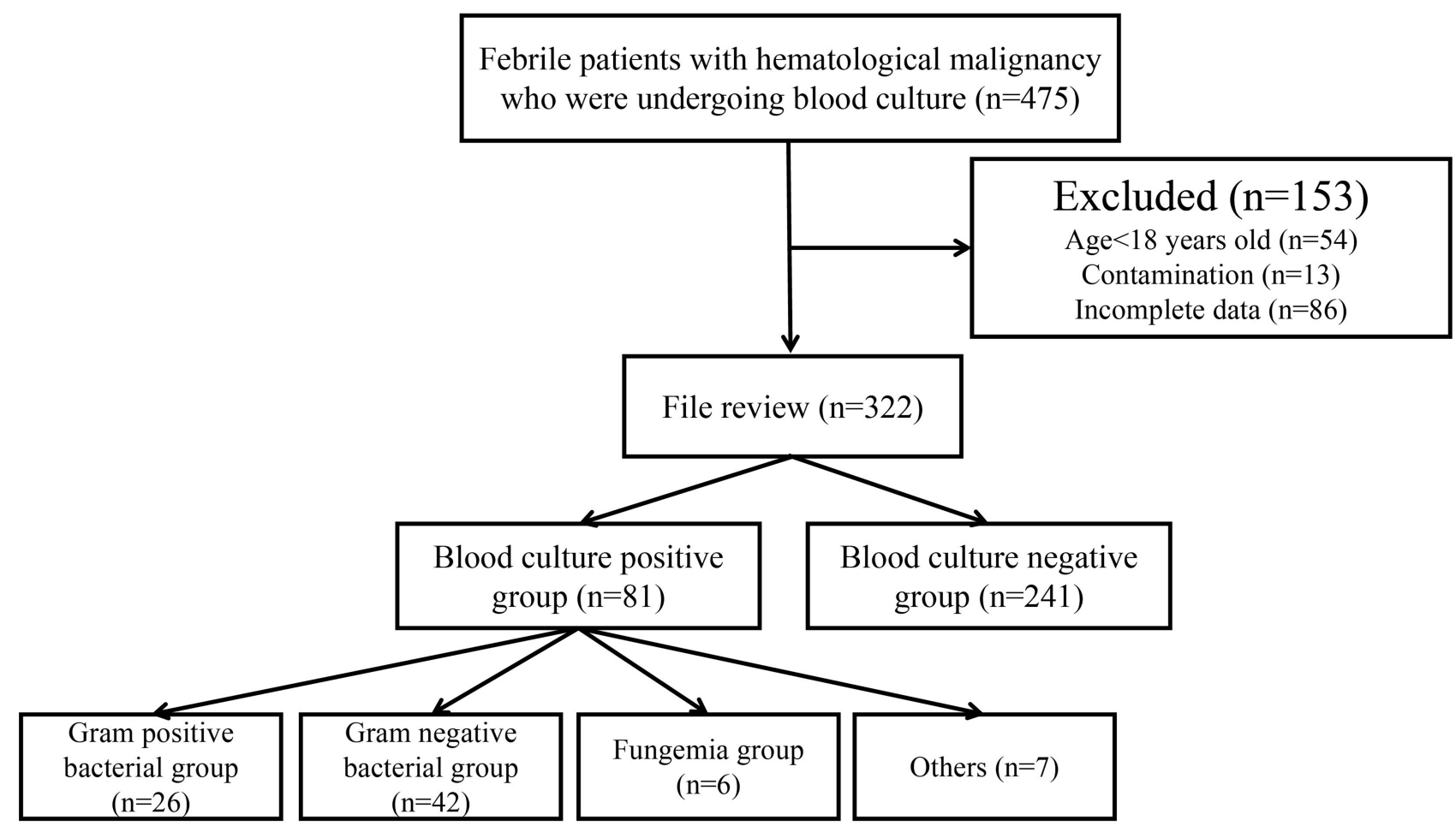

Figure I Flow chart of data collection during the study. 475 blood cultures were obtained; 54 patients were removed because they were under 18 years old; 86 patients were excluded due to incomplete data and 13 because of contamination. The remaining subjects were 322 analyzed in this study. 
Table I Comparison of Clinical Characteristics Between Blood Culture (+) and Blood Culture (-) Group

\begin{tabular}{|l|l|l|l|}
\hline Characteristics & $\begin{array}{l}\text { Blood Culture } \\
(+)\end{array}\left(\begin{array}{l}\text { (n=8 I) } \\
\text { Blood Culture } \\
(-)(\mathbf{n = 2 4 I )}\end{array}\right.$ & P-value \\
\hline $\begin{array}{l}\text { Sex (Male), } \\
\text { no(\%) }\end{array}$ & $49(60.5)$ & $148(61.4)$ & 0.884 \\
\hline $\begin{array}{l}\text { Age, median } \\
\text { (range) }\end{array}$ & $44(21-87)$ & $43(18-91)$ & 0.193 \\
\hline
\end{tabular}

Underlying disease, no\%

\begin{tabular}{|l|l|l|l|}
\hline AML & $35(43.2)$ & $98(40.7)$ & 0.687 \\
\hline ALL & $9(11.1)$ & $43(17.8)$ & 0.154 \\
\hline Lymphoma & $23(28.4)$ & $63(26.1)$ & 0.692 \\
\hline MDS & $4(4.9)$ & $16(6.6)$ & 0.583 \\
\hline MM & $5(6.2)$ & $9(3.7)$ & 0.353 \\
\hline AA & $1(1.3)$ & $7(2.9)$ & 0.685 \\
\hline Others & $4(4.9)$ & $5(2.2)$ & 0.237 \\
\hline
\end{tabular}

Complications, no\%

\begin{tabular}{|c|c|c|c|}
\hline Pneumonia & $37(45.7)$ & $86(35.7)$ & 0.109 \\
\hline Septicemia & $10(12.3)$ & $3(1.2)$ & 0.001 \\
\hline Cystitis & $7(8.6)$ & $25(10.4)$ & 0.652 \\
\hline $\begin{array}{l}\text { Urinary tract } \\
\text { infection }\end{array}$ & $4(4.9)$ & $6(2.5)$ & 0.278 \\
\hline Perianal infection & $2(2.5)$ & $7(2.9)$ & 1.000 \\
\hline Stress ulcer & I (I.2) & $3(1.3)$ & 1.000 \\
\hline Others & II (I3.7) & $36(14.9)$ & 0.765 \\
\hline $\begin{array}{l}\text { No } \\
\text { complications }\end{array}$ & $9(11.1)$ & $75(31.1)$ & 0.001 \\
\hline Diabetes, no\% & & & 0.522 \\
\hline Yes & $9(I I . I)$ & $21(8.7)$ & \\
\hline No & 72 (88.9) & $220(91.3)$ & \\
\hline Smoke, no\% & & & 0.128 \\
\hline Yes & $25(30.9)$ & $54(22.4)$ & \\
\hline No & $56(69.1)$ & $187(77.6)$ & \\
\hline $\begin{array}{l}\text { Days in hospital, } \\
\text { no\% }\end{array}$ & & & 0.891 \\
\hline$\leq 30$ & $36(44.4)$ & $105(43.6)$ & \\
\hline$>30$ & $45(55.6)$ & $136(56.4)$ & \\
\hline
\end{tabular}

Abbreviations: AML, acute myeloid leukemia lymphoma; $A L L$, acute lymphoblastic leukemia; MDS, myelodysplastic syndrome; MM, multiple myeloma; AA, aplastic anemia; Complications, infection in respiratory system, digestive system, abdominal and perianal. of episodes. Because of the blood culture results, antibiotic use was further targeted. The four major antibiotics that were used in the blood culture-positive group were meropenem (25\%), imipenem (23.4\%) cephalosporins (15.6\%), and vancomycin (14.1\%) (Table 2). In the blood culture-negative group, the results were unclear as to which pathogens caused the infection, and empirical medication of antibiotics was implemented, including cephalosporins (33.6\%), imipenem (24.2\%), and meropenem (16.4\%). G- bacteria were more likely to have drug resistance than G+ bacteria (Table 3). Both G- and G+ bacteria were resistant against penicillin and quinolones, and especially E. coli was most resistant against quinolones. Cephalosporin resistance often occurred in Gbacteria. Resistance against tetracyclines, erythromycin, and clindamycin occurred in most G+ bacteria than G- bacteria. Fungi were likely itraconazole-resistant (Table 3).

\section{Risk Factors for BSI in Hematological Malignancies Patients}

In univariate analysis (Table 4), significant variables $(P<0.2)$ (including sex, smoke, complications, WBC, PLT, CRP, PCT, ALB, GLU, IL-6, and D-D) were involved and allowed adjustment to the multivariate analysis. Age, diabetes, inpatient days, ALT, and AST were shown to be insignificant in infection, which might be due to the number of patients or the statistical analysis. Multivariate analysis confirmed six variables were significantly associated with infection: accompanying complications (OR [95\% CI]: 3.459 [1.183-10.109]; $P=0.023)$, PCT (1.29 [1.107-1.503]; $P=0.001)$, ALB (0.885 [0.795-0.92]; $\quad P<0.001), \quad$ GLU (1.297 [1.092-1.539]; $P=0.003), \quad$ IL-6 $\quad(1.048 \quad[1.025-1.07] ; \quad P<0.001) \quad$ and D-D (1.048 [1.025-1.07]; $P<0.001$ ) (Table 5). No such association was observed in sex, smoke, CRP, or PLT in multivariate analysis.

\section{Diagnostic Accuracy of Indicators for BSI Detection}

ROC curves of WBC, PLT, CRP, PCT, IL-6, D-D, ALB, GLU, ALT, and AST levels were plotted to diagnose BSI in 322 febrile episodes (Figure 3). Among these indicators, the AUCs of PCT and D-D were 0.8473 and 0.8613, respectively, indicating higher diagnostic accuracy. Table 6 shows the diagnostic sensitivity and specificity of these indexes with the best cut-off value. When we analyzed WBC, CRP, PCT, IL-6, and D-D in differentiating G- and G+ bacterial infections, IL-6 and D-D all performed better than PCT with an AUC of 0.8182 and 0.8951, respectively. However, 


\section{Distribution of pathogens}

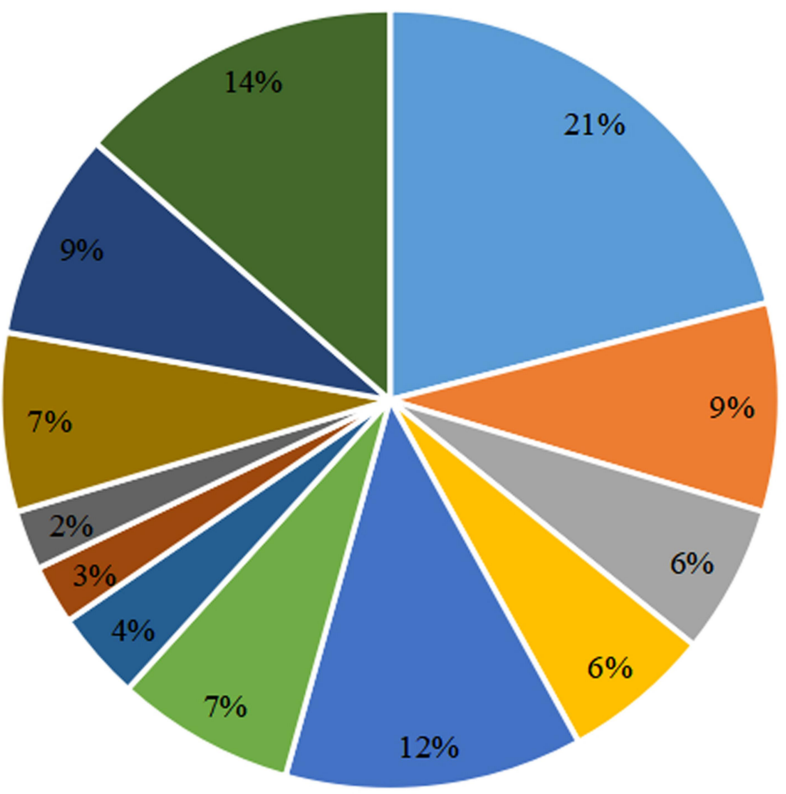

E.coli

K.pneumoniae

P.aeruginosa

Brucella

- S.hominis

S.epidermidis

CNS

S.capitis

S.hemolyticus

- Fungus

- Multi microorganisms $\geq 2$

Others

Figure 2 Distribution of pathogens in blood culture-positive group.

neither WBC nor CRP can effectively differentiate these two groups (Figure 4).

\section{Discussion}

Hematological malignancy patients are more vulnerable to pathogen infection, especially BSI, which leads to higher mortality. The common symptom of infection is fever, though noninfectious febrile episodes are often seen. ${ }^{3}$ It

Table 2 The Use of Antibiotics in Blood Culture-Positive and Blood Culture-Negative Group

\begin{tabular}{|l|l|l|}
\hline Antibiotics Use & $\begin{array}{l}\text { Blood Culture } \\
(+)\end{array}$ & $\begin{array}{l}\text { Blood Cul) } \\
(\mathbf{n = 2 4 I )}\end{array}$ \\
\hline Antibiotics use all & 64 & 128 \\
\hline Meropenem, no\% & $16(25)$ & $21(16.4)$ \\
\hline Imipenem, no\% & $15(23.4)$ & $31(24.2)$ \\
\hline Cephalosporins, no\% & $10(15.6)$ & $43(33.6)$ \\
\hline Moxifloxacin, no\% & $4(6.3)$ & $3(2.3)$ \\
\hline Vancomycin, no\% & $9(14.1)$ & $6(4.7)$ \\
\hline Levofloxacin, no\% & $3(4.7)$ & $3(2.3)$ \\
\hline Teicoplanin, no\% & $0(0)$ & $10(7.8)$ \\
\hline Antifungal drugs, no\% & $2(3.1)$ & $9(7.1)$ \\
\hline Others, no\% & $5(7.8)$ & $2(1.6)$ \\
\hline
\end{tabular}

is important to distinguish infectious from noninfectious patients, which can then be used to provide appropriate and immediate antibiotic therapy. This retrospective study aimed to analyze the differences in clinical characteristics; pathogen distribution; common clinical indicators; diagnostic efficiency; and antibiotic use and resistance between blood culture positive and blood culture negative patients.

We enrolled 322 patients with hematological malignancy, including 81 culture-positive and 241 culturenegative patients. The positive rate of $25.2 \%$ was higher than other reports from different studies, which may be due to our subjects were hematological patients who were more likely to have an infection because of their lowered immunity. ${ }^{11,12}$ AML, lymphoma, and ALL were the most common diseases in the two groups, which are consistent with other studies. ${ }^{13-15}$ In the blood culture-positive group, more G- bacteria (51.9\%) were isolated than $\mathrm{G}+$ bacteria (32.1\%) and fungi (7.4\%), indicating that clinicians should attach great importance to infection by G- bacteria. Meanwhile, G- bacteria were more likely resistant against penicillin, cephalosporins, and quinolones, due to the abuse of broad-spectrum antibiotics, thus bringing a great challenge to treatment. ${ }^{15,16}$ As reported, G+ bacteria were more frequent in some hospitals, even more than G- bacteria, with a high prevalence in clinical laboratory tests. ${ }^{7}$ Antibiotic resistance in $\mathrm{G}+$ bacteria was more common in penicillin, quinolones, tetracyclines, and erythromycin in 
Table 3 Resistance of Antibiotics in Different Pathogens in Blood Culture-Positive Group

\begin{tabular}{|c|c|c|c|c|c|c|c|c|c|}
\hline \multirow{2}{*}{$\begin{array}{l}\text { Antibiotics } \\
\text { Resistance }\end{array}$} & \multicolumn{4}{|c|}{ Gram Negative Bacteria } & \multicolumn{4}{|c|}{ Gram Positive Bacteria } & \multirow{2}{*}{$\begin{array}{l}\text { Fungi } \\
\text { Candida } \\
\text { albicans } \\
\text { (5) }\end{array}$} \\
\hline & $\begin{array}{l}\text { E. coli } \\
\text { (19) }\end{array}$ & $\begin{array}{l}\text { K. pneumoniae } \\
\text { (8) }\end{array}$ & $\begin{array}{l}\text { P. aeruginosa } \\
\text { (6) }\end{array}$ & $\begin{array}{l}\text { Others } \\
\text { (13) }\end{array}$ & $\begin{array}{l}\text { S. hominis } \\
\text { (II) }\end{array}$ & $\begin{array}{l}\text { S. epidermidis } \\
\text { (6) }\end{array}$ & $\begin{array}{l}\text { Other } \\
\text { Staphylococcus } \\
\text { (7) }\end{array}$ & $\begin{array}{l}\text { Others } \\
\text { (5) }\end{array}$ & \\
\hline Penicillins, no\% & $9(47.4)$ & $3(37.5)$ & $3(50)$ & $2(15.4)$ & $8(72.7)$ & $3(50)$ & $2(28.6)$ & I (20) & NA \\
\hline Cephalosporins, no\% & $8(42.1)$ & $2(25)$ & $4(66.7)$ & $4(30.8)$ & I (9.1) & 0 & 0 & 0 & NA \\
\hline Quinolones, no\% & $12(63.2)$ & I (I2.5) & 0 & I (7.7) & $6(54.5)$ & $3(50)$ & $2(28.6)$ & $2(40)$ & NA \\
\hline Sulbactam, no\% & $2(10.5)$ & 0 & 0 & 0 & 0 & 0 & 0 & 0 & NA \\
\hline Aztreonam, no\% & $3(15.8)$ & I (12.5) & 0 & $2(15.4)$ & 0 & 0 & 0 & 0 & NA \\
\hline Gentamicin, no\% & $8(42.1)$ & 0 & 0 & $\mathrm{I}(7.7)$ & 0 & 0 & 0 & 0 & NA \\
\hline Sulfonamides, no\% & $3(15.8)$ & I (12.5) & I (16.7) & 0 & I (9.1) & 1 & 0 & I (20) & NA \\
\hline Furadantin, no\% & 0 & 0 & $2(33.3)$ & I (7.7) & 0 & 0 & 0 & 0 & NA \\
\hline Tetracyclines, no\% & 0 & 0 & 0 & 0 & $5(45.5)$ & I (16.7) & 0 & I (20) & NA \\
\hline Erythromycin, no\% & 0 & 0 & 0 & 0 & 7 & $4(66.7)$ & I (14.3) & 0 & NA \\
\hline Clindamycin,no\% & 0 & 0 & 0 & 0 & 0 & I (16.7) & I (14.3) & 0 & NA \\
\hline Itraconazole, no\% & NA & NA & NA & NA & NA & NA & NA & NA & I (20) \\
\hline
\end{tabular}

the present study. Our results were similar to other reports that empirical antibiotic treatment would increase the drug resistance rate, bringing a considerable burden to both clinical staff and patients. ${ }^{4,7}$ According to blood culture and antibiotic resistance results, meropenem, imipenem and cephalosporins were used more frequently because they were all advanced antibiotics to treat infection. Patients without specific infection often received empirical antibiotic treatment, including these advanced antibiotics that often caused drug resistance.

The multivariate logistical analysis found that patients with complications (such as septicemia), high PCT, GLU, IL-6 and D-D, and low ALB were more likely to have bloodstream infections. It is a common knowledge that hematological malignancy patients are often accompanied by complications due to their immunocompromised status. ${ }^{3}$ Moreover, patients with low ALB concentration are more likely to have lower immunity to resist bacterial infection. ${ }^{17,18}$ The indicators, including PCT, IL-6, and D-D, are inflammation biomarkers to assist in infection diagnosis. Changes in the above factors might indicate the occurrence of infection.

Inflammation biomarkers often serve as assistant indicators in diagnosis. Although there were numerous new biomarkers to aid in diagnosing infection, such as presepsin, CD64 and RANTES, they were all not applied in clinics on a large scale. ${ }^{19-21}$ This study mainly focused on common inflammation biomarkers and explored their function in infection diagnosis. ROC curves were used to investigate the diagnostic efficiency of these biomarkers, including WBC, PLT, CRP, PCT, IL-6, and D-D. WBC is commonly used to indicate inflammation with the level increasing, but we found that WBC counts were lower in the blood culture-positive group. The reason may be that the hematological patients under lower immunity status suffered from chemotherapy or marrow suppression. Meanwhile, WBC's sensitivity and specificity were relatively low, which were insufficient for diagnosing infection. As reported, PLT plays an important role in hemostasis and the protection against bacterial infection. ${ }^{22}$ Platelets have a direct antibacterial defense effect by recognizing microbial antigens and secreting antimicrobial peptides and kinocidins, enhancing innate immune effectors (including complement, neutrophils) and adaptive coordinate immunity (APS, T cells, and B cells). ${ }^{23}$ However, in our study, PLT levels decreased in the blood culture-positive group compared with the blood culture-negative group with an AUC of 0.5803 to differentiate these two groups, and the specificity was too low, which needs further research. 
Table 4 Univariate Analysis of the Risk Factors for Blood Culture Positive and Blood Culture Negative Among 322 Episodes in Patients with Hematologic Malignancies

\begin{tabular}{|c|c|c|c|c|c|}
\hline \multirow[t]{2}{*}{ Variables } & \multicolumn{2}{|c|}{$\begin{array}{l}\text { Blood Culture } \\
(+)(n=8 I)\end{array}$} & \multicolumn{2}{|c|}{$\begin{array}{l}\text { Blood Culture } \\
(-)(n=24 I)\end{array}$} & \multirow[t]{2}{*}{$P$-value } \\
\hline & $\mathbf{N}$ & $\%$ & $\mathbf{N}$ & $\%$ & \\
\hline Age, year & & & & & 0.884 \\
\hline$<60$ & 67 & 82.7 & 213 & 88.4 & \\
\hline$\geq 60$ & 14 & 17.3 & 28 & 11.6 & \\
\hline Sex & & & & & 0.193 \\
\hline Male & 49 & 60.5 & 148 & 61.4 & \\
\hline Female & 32 & 39.5 & 93 & 38.6 & \\
\hline Diabetes & & & & & 0.522 \\
\hline Yes & 9 & II.I & 21 & 8.7 & \\
\hline No & 72 & 88.9 & 220 & 91.3 & \\
\hline Smoke & & & & & 0.128 \\
\hline Yes & 25 & 30.9 & 54 & 22.4 & \\
\hline No & 56 & 69.1 & 187 & 77.6 & \\
\hline Complications & & & & & 0.001 \\
\hline Yes & 72 & 88.9 & 166 & 68.9 & \\
\hline No & 9 & II.I & 75 & 31.1 & \\
\hline Days in hospital & & & & & 0.891 \\
\hline$\leq 30$ & 36 & 44.4 & 105 & 43.6 & \\
\hline$>30$ & 45 & 55.6 & 136 & 56.4 & \\
\hline Variables & \multicolumn{2}{|c|}{$\begin{array}{l}\text { Blood Culture } \\
(+)(n=8 I) \\
\text { (Median } \\
\pm \text { Quantile) }\end{array}$} & \multicolumn{2}{|c|}{$\begin{array}{l}\text { Blood Culture } \\
(-)(n=24 I) \\
\text { (Median } \\
\pm \text { Quantile) }\end{array}$} & $P$-value \\
\hline WBC (10^9/L) & \multicolumn{2}{|c|}{$\begin{array}{l}1.24 \\
(0.27-16.15)\end{array}$} & \multicolumn{2}{|c|}{$2.7 \mid(0.78-62.23)$} & 0.036 \\
\hline PLT (I0^9/L) & \multicolumn{2}{|c|}{$31(19-233)$} & \multicolumn{2}{|c|}{$39(22-339)$} & 0.016 \\
\hline CRP (mg/dL) & \multicolumn{2}{|c|}{8 (4.16-I I.32) } & \multicolumn{2}{|c|}{4.35 (1.75-8.93) } & $<0.001$ \\
\hline PCT (ng/mL) & \multicolumn{2}{|c|}{$2.65(1.1-5.12)$} & \multicolumn{2}{|c|}{$0.23(0.13-0.67)$} & $<0.001$ \\
\hline ALB (g/L) & \multicolumn{2}{|c|}{$\begin{array}{l}31.5 \\
(27.45-35.1)\end{array}$} & \multicolumn{2}{|c|}{$35.3(32.4-39.2)$} & $<0.001$ \\
\hline GLU (mmol/L) & \multicolumn{2}{|c|}{$6.95(5.24-8.99)$} & \multicolumn{2}{|c|}{$5.3(4.75-6.64)$} & $<0.001$ \\
\hline ALT (U/L) & \multicolumn{2}{|c|}{$19.5(9.95-46.4)$} & \multicolumn{2}{|c|}{$\begin{array}{l}18.7 \\
(11.25-31.25)\end{array}$} & 0.475 \\
\hline
\end{tabular}

(Continued)
Table 4 (Continued).

\begin{tabular}{|c|c|c|c|c|c|}
\hline \multirow[t]{2}{*}{ Variables } & \multicolumn{2}{|c|}{$\begin{array}{l}\text { Blood Culture } \\
(+)(n=81)\end{array}$} & \multicolumn{2}{|c|}{$\begin{array}{l}\text { Blood Culture } \\
(-)(n=24 I)\end{array}$} & \multirow[t]{2}{*}{$P$-value } \\
\hline & $\mathbf{N}$ & $\%$ & $\mathbf{N}$ & $\%$ & \\
\hline AST (U/L) & \multicolumn{2}{|c|}{$16.2(10.5-31.7)$} & \multicolumn{2}{|c|}{$17.1(11.65-30.2)$} & 0.679 \\
\hline IL-6 (pg/mL) & \multicolumn{2}{|c|}{32.1 (I2.2-72.4) } & \multicolumn{2}{|c|}{$7.58(4.2-15.22)$} & $<0.001$ \\
\hline $\mathrm{D}-\mathrm{D}(\mu \mathrm{g} / \mathrm{mL})$ & \multicolumn{2}{|c|}{3.1 (I.36-5.37) } & \multicolumn{2}{|c|}{$0.6 I(0.4 \mid-I . I)$} & $<0.001$ \\
\hline
\end{tabular}

CRP, PCT, IL-6, and D-D levels significantly rose in the blood culture-positive group than the blood culturenegative group and performed well in differentiating these two groups. In comparison, IL-6 and D-D outperformed CRP and PCT in differentiating G-/+ groups, with AUC all above 0.8 . CRP and PCT are commonly applied for infection detection. However, CRP concentrations often increase in noninfectious complications and are nonspecific for infection. ${ }^{24}$ PCT is rapidly produced by thyroid gland $\mathrm{C}$ cells and several other types of cells and is stimulated by bacterial endotoxins, lipopolysaccharides, or inflammatory modulators (eg, tumor necrosis factor- $\alpha$, IL-6, IL-1). ${ }^{25}$ PCT reportedly can distinguish infection from non-infection. Yang et al also found that PCT outperformed CRP in separating G- and $\mathrm{G}+$ infection in hematological malignancy patients. ${ }^{2,26}$ IL-6 secreted into the blood initiates division of both $\mathrm{B}$ and $\mathrm{T}$ cells and the generation of antibodies and acute stage proteins during acute stage infections. IL-6 is also involved in the activation of cytokine cascade in sepsis. IL-6 seems helpful in the early assessment of sepsis and is related to disease

Table 5 Multivariate Analysis of the Risk Factors for Infection/ Noninfection Among 322 Episodes in Patients with Hematologic Malignancies

\begin{tabular}{|l|l|l|l|}
\hline \multirow{2}{*}{ Variables } & \multicolumn{3}{|l|}{ Infection/Noninfection } \\
\cline { 2 - 4 } & OR & $95 \%$ CI & P-value \\
\hline Complications & 3.459 & $1.183-10.109$ & 0.023 \\
\hline PCT & 1.29 & $1.107-1.503$ & 0.001 \\
\hline ALB & 0.885 & $0.795-0.92$ & $<0.001$ \\
\hline GLU & 1.297 & $1.092-1.539$ & 0.003 \\
\hline IL-6 & 1.048 & $1.025-1.07$ & $<0.001$ \\
\hline D-D & 1.19 & $1.06-1.335$ & 0.003 \\
\hline
\end{tabular}



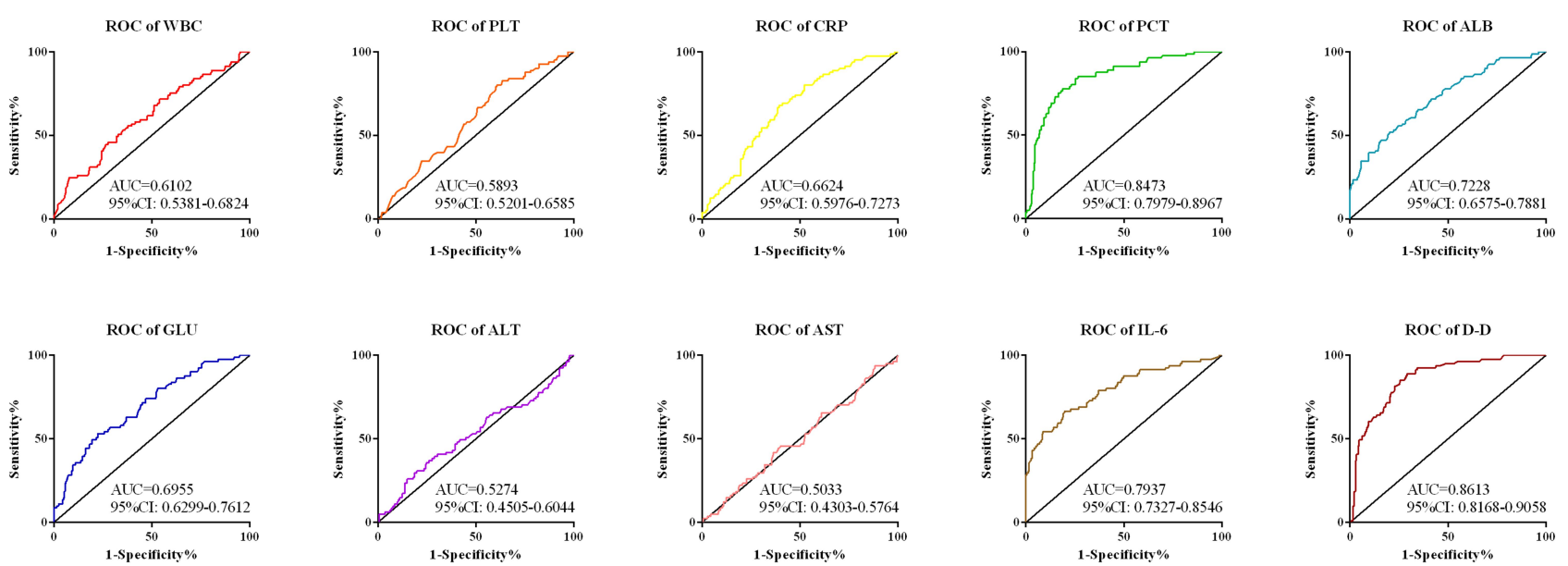

Figure 3 ROC curves of clinical indexes in differentiating blood culture positive and blood culture-negative group. PCT and D-D performed better than other indexes with the AUC all above 0.8

severity. ${ }^{8,9}$ Our results demonstrated that IL-6 performed well in differentiating between infections from noninfections and between $\mathrm{G}-$ and $\mathrm{G}+$ patients. This was also shown to be consistent with previous studies. ${ }^{25,27} \mathrm{D}-\mathrm{D}$, which results from fibrin degradation by fibrinolysis, is commonly used to screen venous thromboembolism clinically. In recent years, the relationship between inflammatory cytokines and biomarkers of the coagulation system has been widely studied, especially in critical patients with sepsis. ${ }^{18,28,29}$ A previous study found that the D-D level increased in pediatric hematological patients with E.coli bacteremia, which may be a clue for physicians to distinguish patients at higher risk for shock and mortality. ${ }^{29}$

Table 6 Sensitivity and Specificity of Parameters in Differentiating Blood Culture Positive from Blood Culture Negative Group

\begin{tabular}{|l|l|l|l|}
\hline Variables & Cut-Off & Sensitivity (\%) & Specificity (\%) \\
\hline WBC (I0^9/L) & 0.895 & 44.44 & 73.44 \\
\hline PLT (I0^9/L) & 58.5 & 80.25 & 39 \\
\hline CRP (mg/dL) & 5.30 & 67.9 & 60.58 \\
\hline PCT (ng/mL) & 0.99 & 77.78 & 81.33 \\
\hline ALB (g/L) & 31.75 & 51.85 & 79.67 \\
\hline GLU (mmol/L) & 6.780 & 53.09 & 77.59 \\
\hline ALT (U/L) & 44.50 & 25.93 & 85.06 \\
\hline AST (U/L) & 20.95 & 41.98 & 63.49 \\
\hline IL-6 (pg/ml) & 18.15 & 66.67 & 80.08 \\
\hline D-D ( $\mu \mathrm{g} / \mathrm{mL})$ & 0.8850 & 88.89 & 70.95 \\
\hline
\end{tabular}

Though our data were all from hematological patients, D-D level increased in the blood culture-positive patients with a sensitivity of $88.89 \%$ and specificity of $70.95 \%$, and performed well in determining $\mathrm{G}-$ or $\mathrm{G}^{+}$patients. However, some reports demonstrated no significant diagnostic value for patients in the early phases of sepsis. ${ }^{30}$ Thus, the function of D-D needs further research.

\section{Study Limitations}

This retrospective study has several limitations. First, it was conducted at a single-center hospital, which might not be generalizable to other settings. Second, we could not compare the laboratory parameters with the clinical course due to insufficient data from patients. Despite these limitations, our study may serve as a reference to guide clinicians in treating patients with a bacterial infection, especially hematological episodes, laying a foundation for the prevention and treatment in the clinic.

\section{Conclusions}

In summary, BSIs were more common in patients with hematological malignancies, of which G- bacteria are more prevalent and often develop antibiotic resistance. Empirical antibiotic use may help prevent infection but may also form drug resistance. Meropenem, imipenem, and cephalosporins show higher activity for the infection. Moreover, our findings demonstrated the risk factors for bacteremia in hematological diseases, including complications, low ALB concentration, high GLU, PCT, IL-6, and D-D levels, which may indicate the occurrence of BSIs. IL-6 and D-D are valuable indicators to aid infection diagnosis. 
ROC of G-/+WBC

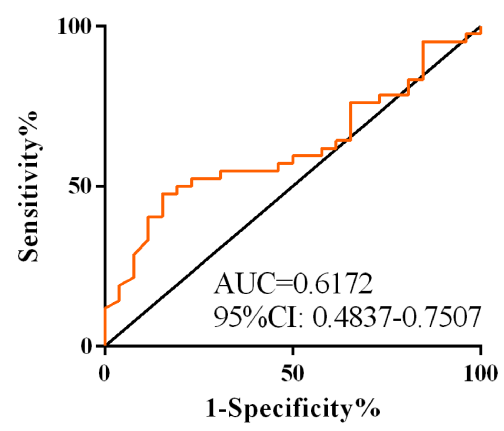

ROC of G-/+CRP

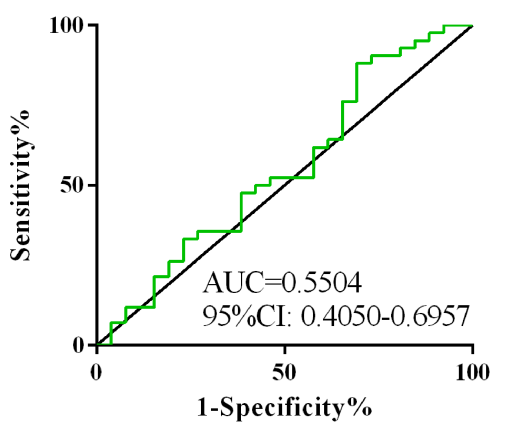

ROC of G-/+PCT

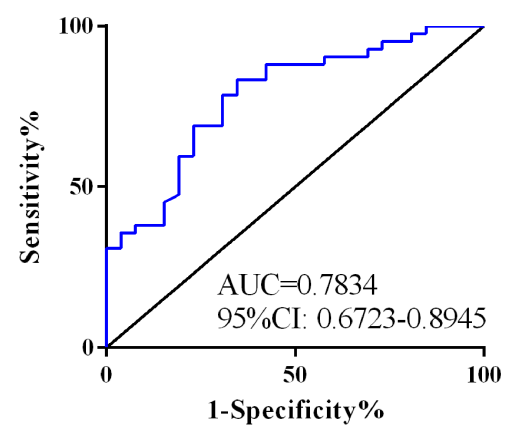

ROC of G-/+IL-6

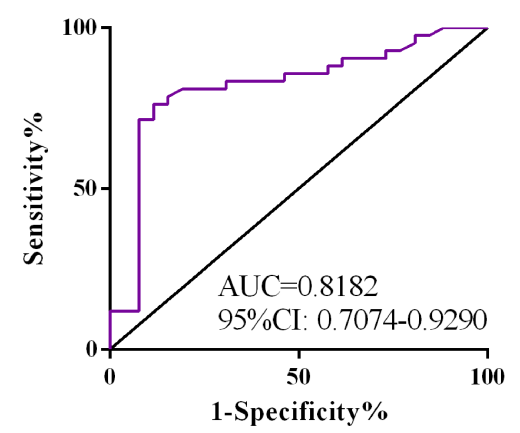

ROC of G-/+ D-D

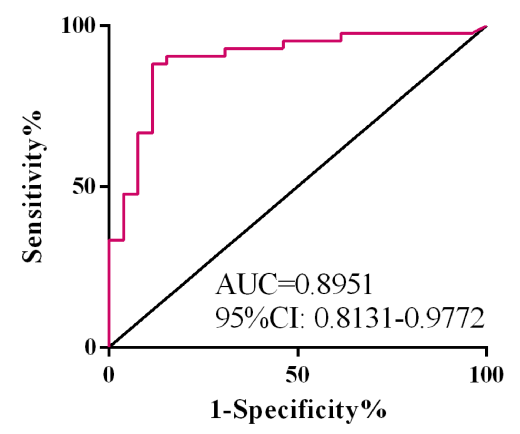

Figure 4 ROC curves of indicators in differentiating Gram- and Gram+ groups. IL-6 and D-D showed excellent diagnosis efficiency in differentiating G- and G+ groups with AUC of 0.8182 and 0.8951 respectively.

Further studies are needed to validate these parameters in patients with a prognosis of different conditions.

\section{Ethics Approval and Consent to Participate}

This study was approved by the Ethical Committee of Chinese PLA General Hospital (No.S2018-207,002). All biological samples were obtained with patients' written informed consent.

\section{Acknowledgments}

The authors would like to thank PLA General Hospital for giving us the repository of clinical data about our study.

\section{Author Contributions}

All authors made substantial contributions to conception and design, acquisition of data, or analysis and interpretation of data; took part in drafting the article or revising it critically for important intellectual content; agreed to submit to the current journal; gave final approval of the version to be published; and agree to be accountable for all aspects of the work.

\section{Funding}

This study was supported by the National Natural Science Foundation of China [grant number 81871731].

\section{Disclosure}

There are no conflicts of interest to declare.

\section{References}

1. Islas-Muñoz B, Volkow-Fernández P, Ibanes-Gutiérrez C, et al. Bloodstream infections in cancer patients. Risk factors associated with mortality. Int $J$ Infect Dis. 2018;71:59-64. doi:10.1016/j. ijid.2018.03.022

2. Yang M, Choi SJ, Lee J, et al. Serum procalcitonin as an independent diagnostic markers of bacteremia in febrile patients with hematologic malignancies. PLoS One. 2019;14(12):e0225765. doi:10.1371/journal. pone. 0225765

3. Russo R, Mendes ET, Levin AS, et al. Bloodstream infection in hematopoietic stem cell transplantation outpatients: risk factors for hospitalization and death. Rev Inst Med Trop Sao Paulo. 2018;61:e3. doi:10.1590/S1678-9946201961003

4. Kagan E, Salgado CD, Banks AL, et al. Peripherally inserted central catheter-associated bloodstream infection: risk factors and the role of antibiotic-impregnated catheters for prevention. Am J Infect Control. 2019;47(2):191-195. doi:10.1016/j.ajic.2018.07.006

5. Ma Y, Wen X, Kong Y, et al. Identification of new peptide biomarkers for bacterial bloodstream infection. Proteomics Clin Appl. 2020;14(2): e1900075. doi:10.1002/prca.201900075 
6. Shao IY, Elkind MSV, Boehme AK. Risk factors for stroke in patients with sepsis and bloodstream infections. Stroke. 2019;50(5):1046-1051. doi:10.1161/STROKEAHA.118.023443

7. El Haddad H, Chaftari A-M, Hachem R, et al. Biomarkers of sepsis and bloodstream infections: the role of procalcitonin and proadrenomedullin with emphasis in patients with cancer. Clin Infect Dis. 2018;67(6):971-977. doi:10.1093/cid/ciy331

8. Fioretto JR, Martin JG, Kurokawa CS, et al. Interleukin-6 and procalcitonin in children with sepsis and septic shock. Cytokine. 2008;43 (2):160-164. doi:10.1016/j.cyto.2008.05.005

9. Hack CE, De Groot ER, Felt-Bersma RJ, et al. Increased plasma levels of interleukin-6 in sepsis. Blood. 1989;74(5):1704-1710. doi:10.1182/blood.V74.5.1704.1704

10. Schwameis M, Steiner MM, Schoergenhofer C, et al. D-dimer and histamine in early stage bacteremia: a prospective controlled cohort study. Eur J Intern Med. 2015;26(10):782-786. doi:10.1016/j. ejim.2015.10.024

11. Shao S-L, Cong H-Y, Wang M-Y, Liu P. The diagnostic roles of neutrophil in bloodstream infections. Immunobiology. 2020;225 (1):151858. doi:10.1016/j.imbio.2019.10.007

12. Zhu S, Kang Y, Wang W, et al. The clinical impacts and risk factors for non-central line-associated bloodstream infection in 5046 intensive care unit patients: an observational study based on electronic medical records. Critical Care (London, England). 2019;23(1):52. doi:10.1186/s13054-019-2353-5

13. Zhang Y, Guo L-Y, Song W-Q, et al. Risk factors for carbapenem-resistant $\mathrm{K}$. pneumoniae bloodstream infection and predictors of mortality in Chinese paediatric patients. BMC Infect Dis. 2018;18(1):248. doi:10.1186/s12879-018-3160-3

14. Lien M-Y, Chou C-H, Lin -C-C, et al. Epidemiology and risk factors for invasive fungal infections during induction chemotherapy for newly diagnosed acute myeloid leukemia: a retrospective cohort study. PLoS One. 2018;13(6):e0197851. doi:10.1371/journal.pone.0197851

15. Yao J-F, Li N, Jiang J. Clinical characteristics of bloodstream infections in pediatric acute leukemia: a single-center experience with 231 patients. Chin Med J. 2017;130(17):2076-2081. doi:10.4103/03666999.213411

16. Marin M, Gudiol C, Ardanuy C, et al. Bloodstream infections in neutropenic patients with cancer: differences between patients with haematological malignancies and solid tumours. $J$ Infect. 2014;69 (5):417-423. doi:10.1016/j.jinf.2014.05.018

17. Zhang Y, Lin J, Shi Q, et al. Diagnostic accuracy of time to first positivity of blood cultures for predicting severe clinical outcomes in children with pneumonia-related bacteremia. $J$ Investig Med. 2020;68:1241-1249. doi:10.1136/jim-2020-001473

18. Yu B, Li X, Chen J, et al. Evaluation of variation in D-dimer levels among COVID-19 and bacterial pneumonia: a retrospective analysis. J Thromb Thrombolysis. 2020. doi:10.1007/s11239-020-02171-y
19. Stoma I, Karpov I, Uss A, et al. Combination of sepsis biomarkers may indicate an invasive fungal infection in haematological patients. Biomarkers. 2019;24(4):401-406. doi:10.1080/1354750X.2019.1600023

20. Stoma I, Karpov I, Uss A, et al. Diagnostic value of sepsis biomarkers in hematopoietic stem cell transplant recipients in a condition of high prevalence of gram-negative pathogens. Hematol Oncol Stem Cell Ther. 2017;10(1):15-21. doi:10.1016/j.hemonc.2016.09.002

21. Stoma I, Karpov I, Milanovich N, et al. Risk factors for mortality in patients with bloodstream infections during the pre-engraftment period after hematopoietic stem cell transplantation. Blood Res. 2016;51 (2):102-106. doi:10.5045/br.2016.51.2.102

22. Sartori MT, Zurlo C, Bon M, et al. Platelet-derived microparticles bearing PF4 and anti-GAGS immunoglobulins in patients with sepsis. Diagnostics (Basel). 2020;10(9). doi:10.3390/diagnostics10090627

23. Hannachi N, Lepidi H, Fontanini A, et al. A novel approach for detecting unique variations among infectious bacterial species in endocarditic cardiac valve vegetation. Cells. 2020;9(8):1899. doi:10.3390/cells9081899

24. Kostic I, Gurrieri C, Piva E, et al. Comparison of presepsin, procalcitonin, interleukin- 8 and $\mathrm{C}$-reactive protein in predicting bacteraemia in febrile neutropenic adult patients with haematological malignancies. Mediterr J Hematol Infect Dis. 2019;11(1):e2019047. doi:10.4084/MJHID.2019.047

25. Wang Q, Wang C, Yang M, et al. Diagnostic efficacy of serum cytokines and chemokines in patients with candidemia and bacteremia. Cytokine. 2020;130:155081. doi:10.1016/j.cyto.2020.155081

26. Fu Y, Chen J, Cai B, et al. The use of PCT, CRP, IL-6 and SAA in critically ill patients for an early distinction between candidemia and Gram positive/negative bacteremia. J Infect. 2012;64(4):438-440. doi:10.1016/j.jinf.2011.12.019

27. Lai L, Lai Y, Wang H, et al. Diagnostic accuracy of procalcitonin compared to $\mathrm{C}$-reactive protein and interleukin 6 in recognizing gram-negative bloodstream infection: a meta-analytic study. Dis Markers. 2020;2020:4873074. doi:10.1155/2020/4873074

28. Hu Q, Fu Y, Tang L. Serum D-dimer as a diagnostic index of PJI and retrospective analysis of etiology in patients with PJI. Clinica Chimica Acta. 2020;506:67-71. doi:10.1016/j.cca.2020.03.023

29. Habe K, Wada H, Higashiyama A, et al. Elevated plasma D-dimer levels in dermatomyositis patients with cutaneous manifestations. Sci Rep. 2019;9(1):1410. doi:10.1038/s41598-018-38108-y

30. Gao Q, Li X, Yu W, et al. Fabrication of mixed-charge polypeptide coating for enhanced hemocompatibility and anti-infective effect. ACS Appl Mater Interfaces. 2020;12(2):2999-3010. doi:10.1021/ acsami.9b19335

\section{Publish your work in this journal}

Cancer Management and Research is an international, peer-reviewed open access journal focusing on cancer research and the optimal use of preventative and integrated treatment interventions to achieve improved outcomes, enhanced survival and quality of life for the cancer patient.
The manuscript management system is completely online and includes a very quick and fair peer-review system, which is all easy to use. Visit http://www.dovepress.com/testimonials.php to read real quotes from published authors. 